Discrete Comput Geom 30:639-648 (2003)

DOI: $10.1007 / \mathrm{s} 00454-003-2856-2$

\title{
The Complexity of Halfspace Area Queries
}

\author{
Stefan Langerman \\ Département d'informatique, Université Libre de Bruxelles, \\ ULB CP212, Av. F. D. Roosevelt 50, B-1050 Bruxelles, Belgium \\ stefan.langerman@ulb.ac.be
}

\begin{abstract}
Given a nonconvex simple polygon $P$ with $n$ vertices, is it possible to construct a data structure which after preprocessing can answer halfspace area queries (i.e., given a line, determine the area of the portion of the polygon above the line) in $o(n)$ time? We answer negatively, proving an $\Omega(n)$ lower bound on the query time of any data structure performing this task. We then consider the offline version of the same problem: given a polygon $P$ with $n$ vertices, and $k$ query lines, we present an algorithm that computes the area of $P$ on both sides of each line in $O\left(k^{2 / 3} n^{2 / 3+\varepsilon}+(n+k)\right.$ polylog $\left.n\right)$ time. Variants of this method allow the query of a collection of weighted polygons with or without holes, and solve several other related problems within the same time bounds.
\end{abstract}

\section{Introduction}

The range query problem can be formulated the following way: given a set $S$ of $n$ points in $\mathbb{R}^{d}$, preprocess $S$ so that, given a range $r \subseteq \mathbb{R}^{d}$, the cardinality of $r \cap S$ can be reported efficiently. There is an extensive literature on this problem (e.g., [8] and [13]), but, surprisingly, very little has been done to generalize it to a more continuous setting. We present several such generalizations. In particular, we consider range queries on the following kinds of objects in $\mathbb{R}^{2}$ :

Polygon $\boldsymbol{P}$. A simple polygon without holes $P$ with $n$ vertices.

Weighted Family of Polygons $\boldsymbol{F}$. A family $F$ of simple polygons with or without holes with a total of $n$ vertices. A weight $w_{i}$ is associated to each polygon $P_{i} \in F$.

Polygon Boundary $\boldsymbol{B}$. A simple polygonal closed chain $B$ with $n$ vertices.

Segments $S$. A set $S$ of $n$ segments. 
Also the following ranges: lines $(l)$ and halfplanes $\left(l^{+}\right.$denotes the halfplane above line l). A halfspace query $l^{+}$on a polygon $P$ or a family of polygons $F$ should report the total area of the intersection $l^{+} \cap P$ or $\sum_{P_{i} \in F} w_{i}$ Area $\left(l^{+} \cap P_{i}\right)$. All other queries should report the total (possibly weighted) length of the intersection. For other results on area partitioning, see [3] and [11].

We will show the following lower bounds:

Theorem 1. Any data structure allowing either of the following:

(a) halfplane queries on a polygon boundary B (or a set $S$ of segments),

(b) line queries on a polygon $P$ (or a family $F$ of polygons),

(c) halfplane queries on a polygon $P$ (or a family $F$ of polygons),

requires $\Omega(n)$ arithmetic operations per query in the straight line program model, regardless of how much memory and preprocessing time is used.

Note that any range query problem on a polygon boundary $B$ is linear time reducible to a query problem on a set $S$ of segments, and a range query problem on a polygon $P$ is linear time reducible to a query problem on a family $F$ of polygons, so we only need to prove the lower bounds for $B$ and $P$, and upper bounds for $S$ and $F$.

The lower bound is particularly relevant when the intersection between the range and the objects has a large number of connected components. For example, for the case (c), if it is known that the boundary $l$ of the query halfplane crosses at most $r$ edges of $P$, some ray shooting data structure could be used to find those $r$ edges that intersect $l$ in $O(r \log n)$, and the area of $P$ above $l$ can then be computed in $O(r)$ time using techniques described in [4] and [7]. This simple method can even be generalized to halfspace volume queries for polyhedra in $\mathbb{R}^{3}$ using the data structure of Iacono and Langerman [12] (and the lower bound clearly holds). Nevertheless, in the worst case $r$ can be $\Theta(n)$, as suggested by the lower bound. Actually, our lower bound proves that a query time of $\Theta(r)$ is the best possible, even if the $r$ segments of $P$ intersected by the query line are known in advance.

Since asking queries one at a time will be expensive in the worst case for any data structure, we consider the offline version of the same problems, and obtain the following result:

Theorem 2. For the range counting problems (a)-(c) from Theorem 1, given the set of objects and $k$ ranges, the answer to all $k$ range queries can be obtained in $O\left(k^{2 / 3} n^{2 / 3+\varepsilon}+\right.$ $(n+k)$ polylog $n)$ time for any small constant $\varepsilon>0$.

Furthermore, for a weighted family of polygons, the polygons are allowed to overlap, in which case the weights of the intersecting regions are cumulative. It is interesting to note that the techniques used could be generalized to allow volume and surface queries in higher dimensions. In the partition graph model of computation that describe geometric divide-and-conquer, Erickson proved a lower bound of $\Omega\left(n \log k+n^{2 / 3} k^{2 / 3}+k \log n\right)$ for solving offline halfspace range queries for points [9] and [10]. This shows that our algorithm is nearly optimal for that model since the algorithm for problem (a) for segments can be used for solving halfspace range queries for points. 


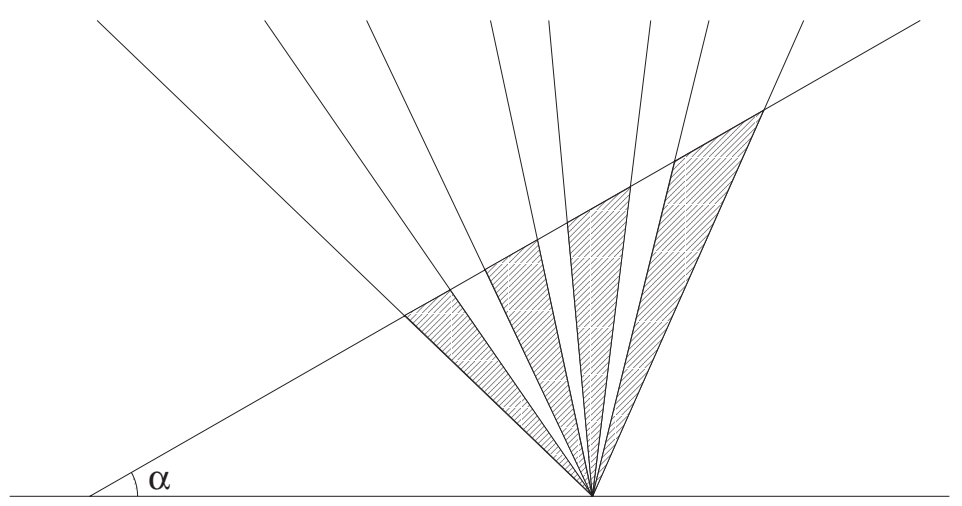

Fig. 1. The fan problem.

While proving the lower bound, we were led to the Fan Problem: given $2 n$ rays from the origin construct a data structure which for a query line $l$ crossing all $2 n$ rays can quickly return the sum of the areas of the $n$ triangles $A_{i}$ bounded by $l$ and the lines $2 i-1$ and $2 i$ (see Fig. 1). We prove an $\Omega(n)$ lower bound on this query time for any data structure, solving an open problem from Jorge Urrutia [1] (also mentioned in [7]). We also give an efficient algorithm for the offline version of this problem.

In the next section we discuss the fan problem, and prove Theorem 1 . We then describe the offline algorithm for case (a) in Section 3, and finish by showing how to modify the algorithm to handle cases (b) and (c).

\section{Lower Bounds and the Fan Problem}

We consider the following problem (see Fig. 2): We are given $n$ lines, with line $L_{i}$ forming an angle $\beta_{i}>0$ with the $x$ axis, and intersecting it at $x=a_{i}$. For a query line $Q$ forming an angle $\alpha>0$ with the $x$ axis and intersecting it at $x=t$, let $l_{i}$ be the signed length of the segment of $L_{i}$ delimited by $Q$ and the $x$ axis. The sign of $l_{i}$ will be

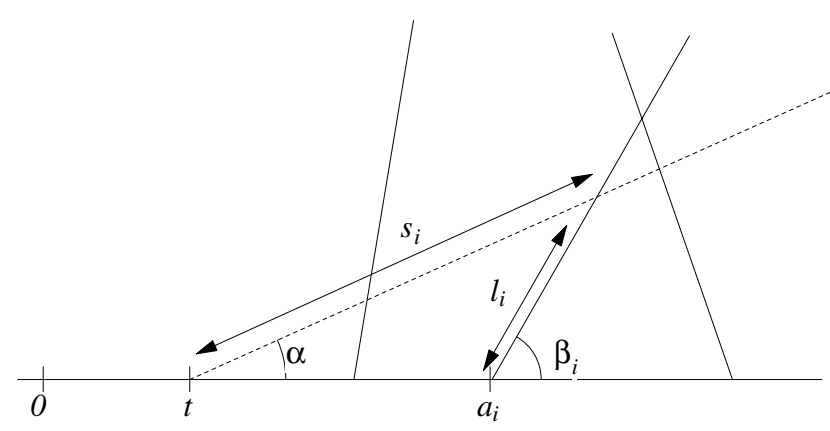

Fig. 2. The general problem. 
positive if the intersection with $Q$ is above the $x$ axis, negative otherwise. Likewise, let $s_{i}$ be the signed length of the segment of $Q$ delimited by $L_{i}$ and the $x$ axis with the same sign convention. Each line has two weights $w_{i}$ and $w_{i}^{\prime}$. Given a query line $Q$, compute quickly $f(Q)=\sum_{i=1}^{n} w_{i} l_{i}$, or $g(Q)=\sum_{i=1}^{n} w_{i}^{\prime} s_{i}$. Using standard trigonometry, we obtain

$$
l_{i}=\frac{\left(a_{i}-t\right) / \sin \beta_{i}}{\cot \alpha-\cot \beta_{i}}
$$

and

$$
s_{i}=\frac{l_{i} \sin \beta_{i}}{\sin \alpha} .
$$

Also note that if $w_{i}=w_{i}^{\prime} \sin \beta_{i}$, then $g(Q)=f(Q) / \sin \alpha$.

We now apply several restrictions to this problem. For example, suppose $n$ is even, $a_{i}=0$, the $\beta_{i}$ are in increasing order, $w_{i}^{\prime}=(-1)^{i+1}$, and, for any query, $\alpha<\beta_{i}$ for all $i$ (and so $t<0$ ). Then $h_{F}(Q)=-\frac{1}{2} t(\sin \alpha) g(Q)$ is exactly the answer to the fan problem described earlier. We restrict further to the case where $t=-1$, let $u=\cot \alpha$, and let $b_{i}=\cot \beta_{i}$, we get

$$
h_{F}(Q)=h_{F}(u)=\frac{1}{2} \sum_{i=1}^{n} \frac{(-1)^{i+1}}{u-b_{i}} .
$$

Note that $h_{F}(u)$ is a rational function in $u$ and that the $b_{i}$ can take arbitrary values. Then any data structure allowing queries for the fan problem will compute $h_{F}(u)$ accurately for an infinite number of $u$ 's.

We now look at another problem. Again, $n$ is even, $a_{i}=0$, and $\beta_{i}$ are in increasing order, but $w_{i}=1$ for all $i$. For any query, $\alpha<\beta_{i}$. This again can be seen as a set of $n$ rays starting at the origin, and the query line $Q$ intersecting all the rays. If $t=-1$, then

$$
h_{S}(Q)=h_{S}(u)=\sum_{i=1}^{n} \frac{1 / \sin \beta_{i}}{u-b_{i}}
$$

is the sum of the lengths of the $n$ segments between the origin and $Q$.

Now, construct a polygon $P$ as follows: Starting with our $n$ rays as above, pick three angles $\alpha_{0}<\alpha_{1}<\alpha_{2}<\beta_{1}$, and let $Q_{j}$ be the query line for angle $\alpha_{j}$ (with $t=-1$ ). Let $p_{i}^{j}$ be the intersection of line $L_{i}$ with $Q_{j}, i=1, \ldots, n$. $P$ (see Fig. 3) will have the sequence of vertices

$$
p_{1}^{0}, p_{2}^{0}, p_{2}^{1}, p_{3}^{1}, p_{3}^{0}, \ldots, p_{2 i-1}^{0}, p_{2 i}^{0}, p_{2 i}^{1}, p_{2 i+1}^{1}, p_{2 i+1}^{0}, \ldots, p_{n}^{0}, p_{n}^{2}, p_{1}^{2}, p_{1}^{0} .
$$

For any query line $Q$ with angle $\alpha, \alpha_{0}<\alpha<\alpha_{1}$, the area of $P \cap Q^{+}$is $A(Q)=$ Area $(P)-h_{F}(Q)+h_{F}\left(Q_{0}\right)$, the total length of $P \cap Q$ is $S(Q)=2 h_{F}(Q) / \sin \alpha$, and the length of the boundary of $P$ above $Q$ is $B(Q)=\operatorname{Boundary}(P)-h_{S}(Q)+$ $h_{S}\left(Q_{0}\right)-S\left(Q_{0}\right)$. From this, we deduce that any data structure that can answer queries of type (a)-(c) (as stated in Theorem 1) will be able to compute $h_{F}(u)$ or $h_{S}(u)$ within the same asymptotic running times, for an infinite number of values of $u$. 


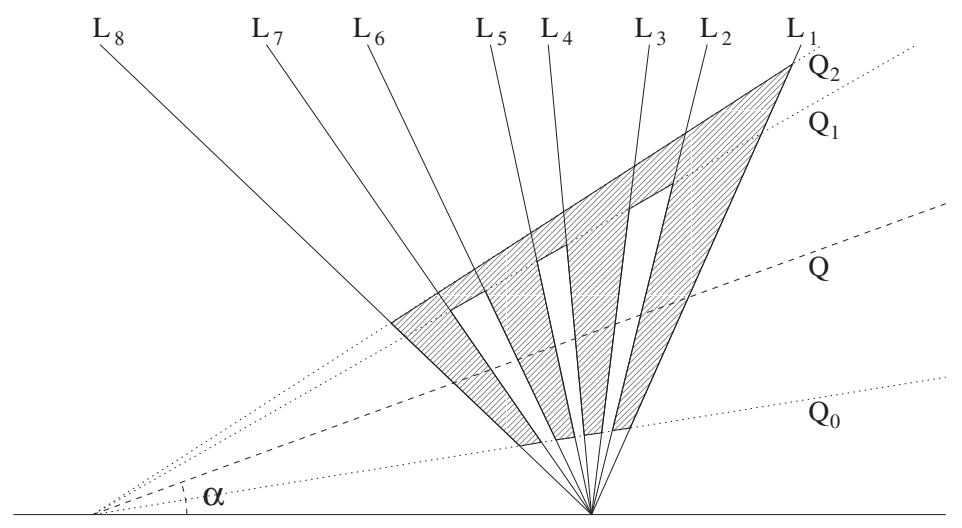

Fig. 3. Polygon $P$.

Thus, in order to prove Theorem 1, we just need to prove the following.

Lemma 3. Given the rational functions $h_{F}(u)$ or $h_{S}(u)$, any data structure allowing queries on the value $h_{F}(u)$ or $h_{S}(u)$ for some u require $\Omega(n)$ arithmetic operations per query, regardless of how much memory and preprocessing are used.

The lemma is proved by application of Motzkin's theorem [15]:

Theorem 4. Let $K$ be an infinite field. If $u, v \in K[x]$ are relatively prime and the leading coefficient of $v$ is 1 , then

$$
L_{+}(u / v) \geq T(u, v)-1, \quad L_{*}(u / v) \geq \frac{1}{2}(T(u, v)-1),
$$

where $L_{+}(f)$ is the minimum number of additions and subtractions, and $L_{*}(f)$ is the minimum number of multiplications and divisions, sufficient to evaluate $f$, where operations not involving $x$ are regarded as costless. $T(u, v)$ is the degree of transcendence of the set of coefficients of $u$ and $v$ over the primefield of $K$.

For computing $h_{F}(x) \in k\left(b_{1}, \ldots, b_{n}, x\right)$, we enlarge the groundfield $k$ to the extention field $K=k\left(b_{1}, \ldots, b_{n}\right)$. Note that the set of coefficients of $h_{F}(x) \in K(x)$ written as a quotient of polynomials has degree of trancendence $\Omega(n)$ and apply Theorem 4 . Repeat this argument for $h_{S}(x)$. This completes the proof of Lemma 3.

\section{Offline Queries}

\subsection{Ray Problem}

We first study the case of batched queries of type (a): given a set $S=\left\{s_{1}, \ldots, s_{n}\right\}$ of $n$ segments in $\mathbb{R}^{2}$ and $k$ lines $Q_{1}, \ldots, Q_{k}$, determine for each line $Q_{i}$ the sum of the lengths of the segments $s_{j} \cap Q_{i}^{+}$. Note that the problem for polygon boundaries can be 
reduced to this one by just choosing $S$ to be the set of edges of the polygon. We assume without loss of generality that all the elements of $S$ are strictly contained in the positive quadrant in the $x$ and $y$ coordinates.

In order to simplify this problem, we view each segment as the difference of two rays pointing downwards (i.e., crossing the $x$ axis). For example, if segment $(a, b)$ has endpoint $a$ of smaller $y$ coordinate than $b$, it will be replaced by the ray starting at $b$ extending the segment downwards, with weight 1 , and a second segment of same support and direction, but starting at $a$, and with a weight of -1 . The problem is then reduced to the following: given a set of rays $R=\left\{r_{1}, \ldots, r_{n}\right\}$, with weights $w_{1}, \ldots, w_{n}$, and $k$ lines $Q_{1}, \ldots, Q_{k}$, determine for each line $Q_{i}$ the sum of the lengths of the segments $r_{j} \cap Q_{i}^{+}$multiplied by their weights $w_{j}$. Computing the length of $r_{j} \cap Q_{i}^{+}$is the same as computing the (signed) length of the segment of $r_{j}$ delimited by $Q_{i}$ and the $x$ axis, and subtracting that number from the length of $r_{j}$ above the $x$ axis. However, this is exactly the problem discussed in Section 2, so we get the following:

Lemma 5. Given $n$ rays $r_{1}, \ldots, r_{n}$ with weights $w_{1}, \ldots, w_{n}$, all intersecting some query line $Q$. The weighted sum of the lengths of $r_{j} \cap Q^{+}$can be computed by the rational function $C-f(Q)$ where $f$ is as described in Section 2 and $C$ is some constant. This rational function is the quotient of two polynomials of degree $n$.

Note that the natural form (quotient of two polynomials) of this rational function can be computed in $O\left(n \log ^{2} n\right)$ time using divide and conquer and Fast Fourier Transform. We can also factor out the parameter $t$ to obtain an expression of the form $p_{1}(u) / q_{1}(u)+$ $t\left(p_{2}(u) / q_{2}(u)\right)$. The next step is to batch the queries: it is known that a polynomial of degree $n$ can be evaluated for $k$ values in $O\left((n+k) \log ^{2} n\right)$ using FFT (see, e.g., [2]). We then conclude that our rational function can be evaluated $k$ times within the same time bounds, and we get:

Lemma 6. Given a set of rays $R=\left\{r_{1}, \ldots, r_{n}\right\}$, with weights $w_{1}, \ldots, w_{n}$, and $k$ lines $Q_{1}, \ldots, Q_{k}$ each intersecting all $n$ rays, we can compute for each line $Q_{i}$ the sum of the lengths of the segments $r_{j} \cap Q_{i}^{+}$multiplied by their weights $w_{j}$, in $O\left((n+k) \log ^{2} n\right)$.

However, this assumes that each query line intersects all rays. For the general case, we will have to do some more work.

\subsection{Partitioning}

The idea of this algorithm is similar to the one used by Matoušek in [14] for Hopcroft's problem. We use the notion of cuttings. A cutting $\Xi$ is a collection of closed $d$-dimensional simplices with disjoint interiors, covering $\mathbb{R}^{d}$ (a simplex is the nonempty intersection of $d+1$ halfspaces in $\mathbb{R}^{d}$ and may be unbounded). Let $H$ be a set of $n$ hyperplanes in $\mathbb{R}^{d}$, and write $H_{\Delta}$ for the set of hyperplanes in $H$ intersecting the interior of the 
simplex $\Delta . \Xi$ is an $\varepsilon$-cutting for $H$ if, for any simplex $\Delta \in \Xi,\left|H_{\Delta}\right| \leq \varepsilon n$. We use the following:

Theorem 7. For any collection of $n$ hyperplanes in $\mathbb{R}^{d}$ and a parameter $s \leq n$, there exists a $(1 / s)$-cutting of size $O\left(s^{d}\right)$ [6]. Such a cutting can be computed in $O\left(n s^{d-1}\right)$ time by a randomized [6] or deterministic algorithm [5].

By an $(n, k)$-problem, we mean the problem of computing the weighted sum of lengths for $n$ rays and $k$ query lines. We first show how to solve an $(n, k)$-problem by combining many smaller-sized problems.

Lemma 8. An (n, k)-problem can be solved in time $O\left(\left(n+k s^{2}\right) \log ^{3} n\right)$ plus the time needed for solving $O\left(s^{2}\right)\left(n / s^{2}, k / s\right)$-problems for some given parameter $1<s<$ $\min (\sqrt{n}, k)$.

Proof. We are given a set of rays $R=\left\{r_{1}, \ldots, r_{n}\right\}$, with weights $w_{1}, \ldots, w_{n}$, and a set $H$ of $k$ lines $Q_{1}, \ldots, Q_{k}$. For this discussion, we rotate the plane so that all rays are pointing to the positive $x$ direction. We then have the following:

Fact 9. A ray starting at point $p$ pointing to the positive $x$ direction with slope $m_{r}$ intersects a line $l$ of slope $m_{l}$ if and only if $l$ is above $p$ and $m_{r}>m_{l}$, or $l$ is below $p$ and $m_{r}<m_{l}$.

We first construct a cutting $\Xi$ of size $O\left(s^{2}\right)$ for the lines in $H$, along with the subset of lines $H_{\Delta}$ intersecting the interior of each simplex $\Delta$. Then locate the starting point of each ray in $R$ in the cutting. This takes time $O(k s+n \log s)$. Subdivide the simplices further to ensure that each simplex contains at most $n / s^{2}$ starting points of rays. We now have a $(1 / s)$-cutting $\Xi^{\prime}$, still of size $O\left(s^{2}\right)$ and for each simplex $\Delta \in \Xi^{\prime}$ we know the set $H_{\Delta}$ of lines intersecting the interior of $\Delta$, and the set $R_{\Delta}$ of rays whose starting point is in $\Delta ;\left|H_{\Delta}\right| \leq k / s$ and $\left|R_{\Delta}\right| \leq n / s^{2}$.

The set of intersections between a ray and a query line can be divided into groups. For each simplex $\Delta$, we consider first the intersections between the rays in $R_{\Delta}$ and the query lines not intersecting the interior of $\Delta$, and then the intersections between rays in $R_{\Delta}$ and lines in $H_{\Delta}$. For each simplex $\Delta$, we construct a balanced binary tree for the slopes of all rays in $R_{\Delta}$, i.e., the root of the tree will contain all rays, its left child will contain the $\left\lfloor\left|R_{\Delta}\right| / 2\right\rfloor$ rays with the smallest slopes, the right child the $\left\lceil\left|R_{\Delta}\right| / 2\right\rceil$ rays with the largest slopes, and so on recursively. For each line $Q_{i} \in H-H_{\Delta}$ and using Fact 9 we query the tree, finding all rays in $R_{\Delta}$ that intersect $Q_{i}$ as a disjoint union of $O\left(\log \left(n / s^{2}\right)\right)$ sets corresponding to at most one node at each level of the tree. Mark each of these nodes with a pointer to $Q_{i}$. Using Lemma 6, we can compute for each tree node $j$ and for each line $Q_{i}$ stored in that node the weighted sum of the lengths of the portions of rays in node $j$ above $Q_{i}$, in $O\left(\left(a_{j}^{\prime}+r_{j}^{\prime}\right) \log ^{2} r_{j}^{\prime}\right)$ time, where $r_{j}^{\prime}$ is the number of rays 
stored in node $j$, and $a_{j}^{\prime}$ is the number of $Q_{i}$ 's stored in node $j$. The total running time will then be

$$
O\left(\sum_{\text {node }_{j}}\left(a_{j}^{\prime}+r_{j}^{\prime}\right) \log ^{2}\left(r_{j}^{\prime}\right)\right) \leq O\left(\sum_{\text {node }_{j}}\left(a_{j}^{\prime}+r_{j}^{\prime}\right) \log ^{2}(n)\right) .
$$

Since every ray only appears once in each of the $O(\log n)$ levels of the binary tree, $\sum_{\text {node } j} r_{j}^{\prime}=O\left(\left(n / s^{2}\right) \log n\right)$. Because each $Q_{i}$ is contained in at most one node at each of the $O(\log n)$ levels of the tree, $\sum_{\text {node } j} a_{j}^{\prime}=O(k \log n)$. The cost for computing the partial results for the rays in $R_{\Delta}$ and the lines in $H-H_{\Delta}$ is $O\left(\left(n / s^{2}+k\right) \log ^{3} n\right)$ and the total cost for all $\Delta$ is $O\left(\left(n+k s^{2}\right) \log ^{3} n\right)$.

After this, we still have to compute the partial results for the rays in $R_{\Delta}$ and the lines in $H_{\Delta}$, but this can be done by solving an $\left(n / s^{2}, k / s\right)$-problem.

We now prove a dual version of this lemma.

Lemma 10. An (n, k)-problem can be solved in time $O\left(\left(n s^{2}+k\right) \log ^{2} n\right)$ plus the time needed for solving $O\left(s^{2}\right)\left(n / s, k / s^{2}\right)$-problems for some given parameter $1<s<$ $\min (n, \sqrt{k})$.

Proof. We are given a set of rays $R=\left\{r_{1}, \ldots, r_{n}\right\}$, with weights $w_{1}, \ldots, w_{n}$, and a set $H$ of $k$ lines $Q_{1}, \ldots, Q_{k}$. Apply the standard duality transform, which maps a point $(a, b)$ to a line $y=a x+b$, and a line $y=m x+c$ to a point $(-m, c)$. This transformation preserves incidence and above/below relations, and a ray starting at point $p$ will be mapped to the double wedge formed by the dual line corresponding to $p$, and a vertical line. This forms a set $G$ of $2 n$ lines. Construct a $(1 / s)$-cutting $\Xi$ for $G$ of size $O\left(s^{2}\right)$, along with, for each simplex $\Delta$, the subset $G_{\Delta}$ of lines intersecting $\Delta$. We have $\left|G_{\Delta}\right| \leq n / s$, and for all the rays $r_{i}$ in $R$ whose corresponding (dual) lines are not in $G_{\Delta}$, either all the points in $\Delta$ transform to lines crossing $r_{i}$ or none of them do. For each point dual to a line in $H$, find the simplex $\Delta$ that contains it. This takes time $O(n s+k \log s)$. As in the previous lemma, subdivide the simplices further so that no simplex contains more than $O\left(k / s^{2}\right)$ points (dual to the lines in $H$ ). Once again, we will first compute the subproblems for query lines corresponding to points in some $\Delta$, and rays corresponding to lines not intersecting $\Delta$. This, for each $\Delta$, will correspond to a subproblem with $n$ rays and $k / s^{2}$ query lines all intersecting, and can be solved in $O\left(\left(n+k / s^{2}\right) \log ^{2} n\right)$ using Lemma 6 , and for all $\Delta$ in $O\left(\left(n s^{2}+k\right) \log ^{2} n\right)$. The remaining subproblems are for query lines corresponding to points in $\Delta$ and rays whose corresponding lines intersect $\Delta$. They are the $O\left(s^{2}\right)\left(n / s, k / s^{2}\right)$-problems.

Alternating the two lemmas, we obtain the following recurrence for the running time of this algorithm:

$$
T(n, k) \leq O\left(s^{2}(n+k) \log ^{3} n\right)+O\left(s^{4}\right) T\left(n / s^{3}, k / s^{3}\right)
$$


and for any given constant $\varepsilon>0$, the constant $s$ can be chosen so that $T(n, k)=$ $O\left(k^{2 / 3} n^{2 / 3+\varepsilon}\right)$, if $n>\sqrt{k}$ and $k>\sqrt{n}$, and so, in general, the overall running time is

$$
O\left(n^{2 / 3} k^{2 / 3+\varepsilon}+(n+k) \text { polylog } n\right) .
$$

This completes the proof of Theorem 2 for queries of type (a).

\section{Queries (b) and (c)}

In the previous section we described how to batch halfspace queries for a set of segments. Case (b) of the line queries can be treated similarly, by turning it into a ray problem, but using the rational function $g(Q)$ (as described in Section 2) instead of $f(Q)$ ). In the remainder of this section, we concentrate on case (c) for a family $F$ of polygons.

For halfspace queries on a family of polygons $F$, suppose we associate to every edge $e$ the signed area $a(e)$ of the trapezoid defined by $e$ and its projection on the $x$ axis, positive if the interior of a polygon is below $e$, and negative otherwise. Then $\sum_{e \in F} a(e)$ is the total area of the family of polygons. In order to perform a halfspace query $Q^{+}$on this family, we will need to

(i) sum the signed area for the edges above the query line $Q$, some of them truncated, and

(ii) add the signed area of the newly added edges for the upper part of the polygons in $F$ (note that all the new edges lie on $Q$ ).

As in Section 3.1, we will turn every edge into two rays. The value of $a(e)$ is the difference between the areas of the triangles below the rays associated with $e$. The area of such a triangle for line $L_{i}$ and query $Q$ is $\frac{1}{2}\left(l_{i} \sin \beta_{i}\right)\left(l_{i} \cos \beta_{i}\right)$ and the total for (i) is

$$
\sum \frac{w_{i}}{2}\left(l_{i}^{2} \sin \beta_{i} \cos \beta_{i}\right)
$$

for some weights $w_{i}$, and is a rational function of degree $O(n)$. Thus, the technique described in Section 3 can be applied, and part (i) of the query can be computed within the bounds of Theorem 2 .

As noted before, all newly added edges lie on the query line $Q$. Thus, the corresponding signed areas are again the difference between the area of two triangles below $Q$. Each such triangle area can be expressed as

$$
\frac{1}{2}\left(a_{i}-t+l_{i} \cos \beta_{i}\right)\left(l_{i} \sin \beta_{i}\right)
$$

and the same techniques can once more be used.

To solve the weighted problem, we just multiply the weight of each triangle by the weight of the corresponding polygon.

\section{Acknowledgments}

I thank John Iacono, William Steiger, and Mario Szegedy for helpful discussions, and Micha Sharir whose comments allowed me to improve the upper bound in Theorem 2. 


\section{References}

1. Open problems session. Presented at Twelfth Computational Geometry Day, 1997.

2. A. V. Aho, J. E. Hopcroft, and J. D. Ullman. The Design and Analysis of Computer Algorithms. AddisonWesley, Reading, MA, 1974.

3. K.-F. Böhringer, B. Donald, and D. Halperin. On the area bisectors of a polygon. Discrete Comput. Geom., 22(2):269-285, 1999.

4. R. Boland and J. Urrutia. Polygon area problems. In Proceedings of 12th Canadian Conference on Computational Geometry, pages 159-162, 2000.

5. B. Chazelle. Cutting hyperplanes for divide-and-conquer. Discrete Comput. Geom., 9(2):145-158, 1993.

6. B. Chazelle and J. Friedman. A deterministic view of random sampling and its use in geometry. Combinatorica, 10(3):229-249, 1990.

7. F. Contreras-Alcalá. Cutting polygons and a problem on illumination of stages. M.Sc. thesis, Dept. Computer Science, University of Ottawa, Ottawa, 1998.

8. M. de Berg, M. van Kreveld, M. Overmars, and O. Schwarzkopf. Computational Geometry: Algorithms and Applications. Springer-Verlag, Berlin, 1997.

9. J. Erickson. New lower bounds for Hopcroft's problem. Discrete Comput. Geom., 16:389-418, 1996.

10. J. Erickson. Space-time tradeoffs for emptiness queries. SIAM J. Comput., 29(6):1968-1996, 2000.

11. R. Guàrdia and F. Hurtado. On the equipartitions of convex bodies and convex polygons. In Abstracts 16th European Workshop on Computational Geometry, pages 47-50. Ben-Gurion University of the Negev, 2000.

12. J. Iacono and S. Langerman. Volume queries in polyhedra. In Proceedings of Japan Conference on Discrete and Computational Geometry, pages 156-159, 2000.

13. J. Matoušek. Efficient partition trees. Discrete Comput. Geom., 8:315-334, 1992.

14. J. Matoušek. Range searching with efficient hierarchical cuttings. Discrete Comput. Geom., 10(2):157$182,1993$.

15. V. Strassen. Algebraic complexity theory. In J. van Leeuwen, editor, Algorithms and Complexity, volume A of Handbook of Theoretical Computer Science, chapter 11, pages 633-672. MIT Press, Cambridge, MA, 1990.

Received February 27, 2002, and in revised form June 13, 2002. Online publication September 1, 2003. 